\title{
Active conditioning of ASDEX Upgrade tungsten plasma- facing components and discharge enhancement through boron and boron nitride particulate injection
}

\author{
R. Lunsford ${ }^{1}$, V. Rohde ${ }^{2}$, A. Bortolon ${ }^{1}$, R. Dux ${ }^{2}$, A. Herrmann ${ }^{2}$, A. Kallenbach ${ }^{2}$, R. M. McDermott ${ }^{2}$, \\ P. David ${ }^{2}$, A. Drenik ${ }^{2}$, F. Laggner ${ }^{1}$, R. Maingi ${ }^{1}$, D. K. Mansfield ${ }^{1}$, A. Nagy ${ }^{1}$, R. Neu ${ }^{2}$, E. Wolfrum ${ }^{2}$, \\ and the ASDEX Upgrade team \\ ${ }^{I}$ Princeton Plasma Physics Laboratory, 100 Stellarator Rd, Princeton, NJ 08543 USA \\ ${ }^{2}$ Max-Planck-Institut für Plasmaphysik, Boltxmannstr. 2, , 85748 Garching, Germany \\ Email: rlunsfor@pppl.gov
}

\begin{abstract}
The injection of boron $(\mathrm{B})$ and boron nitride $(\mathrm{BN})$ powders into ASDEX Upgrade (AUG) H-mode discharges have demonstrated effective control of tungsten influx in low density/collisionality operational regimes, similar to conventional boronization methods. Sub-mm powder particles are gravitationally accelerated into the upper edge of a lower single null H-mode plasma with a boundary shape roughly conforming to the shape of the poloidal midplane limiters. Visible spectroscopy measurements at one of the outer limiter showed increases in both B and $\mathrm{N}$ signal levels, as well as elevated B levels in the divertor, and an increase in total radiated power by greater than a factor of 2 during BN injection. Globally the BN injection improved energy confinement by $10-20 \%$, associated with improvements in pedestal performance similar to gaseous $\mathrm{N}$ injection. Following conditioning discharges with B powder injection, three low gas-fueling discharges with magnetic perturbations for ELM suppression were successfully conducted. These first results suggest that the application of $\mathrm{B}$ containing powders can be used to both improve plasma performance in real-time, and to improve overall wall conditions for subsequent discharges.
\end{abstract}




\section{INTRODUCTION}

Conditioning of the primary plasma facing components (PFCs) has been found to be essential in maximizing operational space and discharge reproducibility in fusion research devices[1-3]. The periodic application of wall conditioning techniques such as RF conditioning, baking, glow discharge cleaning, and surface deposition of evaporative coatings (boronization, lithiumization, siliconization) lead to advancements in plasma performance[4], and the utilization of pre-operative conditioning is now widely accepted as desirable to establish stable discharges free from high- $\mathrm{Z}$ impurity contamination. In addition, present-day tokamaks $[5,6]$ often use low- $Z$ wall conditioning to either improve access to low collisionality regimes and/or improve energy confinement through control of hydrogenic recycling from PFCs. This includes previous experiments utilizing the controlled introduction of lithium aerosol to examine intra-shot and long term conditioning effects on EAST[7], NSTX[8], and DIII-D[9]

As long pulse devices like LHD, EAST, KSTAR, W7X and others push the limits of their operational space, there exists the possibility that the extended plasma durations and elevated power throughputs will provide an integral wall flux which will degrade wall conditions on a programmatically challenging timescale. As such it is possible that experimental operations may benefit from real-time wall conditioning to maintain optimum wall conditions between discharges or to reapply degraded coatings during discharges if required. To that end the Impurity Powder Dropper (IPD) [10] has been developed to provide in-situ particulate application to determine if this impurity delivery method can be used as a wall conditioning technique.

The injection of boron (B) and boron nitride (BN) powders into AUG conditioning discharges is shown in this paper to effectively control tungsten influx in subsequent AUG low density/collisionality H-modes, similar to conventional boronization methods. In these experiments, B and BN were separately and controllably injected via gravity into H-mode discharges. Total radiated power and plasma energy confinement increased during $\mathrm{BN}$ injection, in a manner qualitatively similar to $\mathrm{N}$ gas injection. In addition, pure 
B injection provided compelling evidence of post-shot residual wall conditioning, allowing access to low collisionality $\mathrm{H}$-modes that otherwise require a recent conventional boronization. These first results hold the promise of rapid wall reconditioning and the possibility to operate with more stable and uniform wall conditions between routine boronizations in AUG. In the remainder of this paper we will describe the effects of standard boronizations on AUG, describe the Impurity Powder Dropper (IPD), and examine the effects of particulate injection into AUG H-Modes.

\section{WALL CONDITIONING IN AUG}

The plasma facing surfaces at ASDEX Upgrade[11, 12] have undergone a stepwise conversion from carbon PFCs to full tungsten PFCs. Mitigation of the $\mathrm{W}$ influx and access to high H-mode energy confinement were achieved through the use of elevated input powers, ELM pacing and ECRH central heating, with additional gas puffing to enhance the ELM frequency and reduce W sputtering[13, 14]. For a Type 1 ELMy Hmode discharge without the beneficial effects of a recent boronization, ELM frequencies greater then $100 \mathrm{~Hz}$ are required to maintain discharge purity. These elevated ELM frequencies also flush $\mathrm{W}$ influx from the edge-pedestal region before the impurities degrade core performance, achieving a stable balanced $\mathrm{W}$ content.

To arrest the incursion of impurities AUG began a series of periodic boronizations comprised of the injection of $10 \%$ concentrations of $\mathrm{B}_{2} \mathrm{D}_{6}$ gas and $90 \%$ He into helium glow discharges[15]. The regular application of a 4 hour boronization has been found to deposit approximately $8 \mathrm{~g}$ of boron in a uniform $30 \mathrm{~nm}$ thickness on the main chamber walls. After boronizations onto the full metal wall a strong reduction in both residual trace carbon and oxygen levels was observed, although the carbon returned to roughly half of the pre-boronization levels within a series of 30-40 high power discharges. As a consequence of the reduced impurity levels the overall plasma radiation level dropped and power exhaust at the outer target was observed to increase. 
A primary effect of the boronization coatings is mitigation of the $\mathrm{W}$ influx without the need for supplemental ELM frequency enhancement through deuterium gas injection. This then enables access to low plasma density and collisionality regimes. These effects are transitory on timescales similar to the resurgence of trace carbon impurity levels mentioned previously. Therefore, it is of programmatic interest for AUG to have a means to reduce the $\mathrm{W}$ influxes to allow extended operations in this low collisionality regime. One such scenario where low density operation is critical is the utilization of resonant magnetic perturbations (RMP) to suppress ELMs[16], as will be discussed in section 5. In addition, it has been seen that ICRF heated discharges are particularly susceptible to enhanced impurity influx, due to the sheath-rectified electric fields at the antenna limiters $[17,18]$. After a boronization, more favorable conditions, most specifically a reduced $\mathrm{W}$ level, have been observed at these limiter locations suggesting a protective boron coating has been overlaid.

The decreased impurity content present in AUG after boronization means that divertor power loading must be closely monitored for routine operation. Nitrogen is the most commonly utilized radiative impurity in AUG, providing divertor cooling while maintaining or even improving energy confinement, and reducing ELM size. Langmuir probe analysis of nitrogen seeded discharges have shown[19] a reduced outer divertor electron temperature (Te), and partial detachment of the outer strike point and full detachment of the inner target. Nitrogen gas puffing was also successful in recovering the performance losses observed with the transition to full metal walls in AUG[20]. We also note that there is short-term wall loading after nitrogen gas injection, and that discharges following those with active $\mathrm{N}$ seeding showed nitrogen levels approximating $50 \%$ of the seeded discharge. With gaseous nitrogen seeding the higher radiation level that was previously present with carbon walls can be reproduced, albeit with a different spatial distribution. This is partially due to the localized source and entrainment into the scrape off layer flows, as discussed in section 4.1. Finally, disruptions with metal walls are less radiative than those that occur within carbon PFC devices, again due to the lower level of intrinsic impurity radiation. As a result massive gas injection with $\mathrm{Ar} / \mathrm{D}_{2}$ mixtures is required to mitigate mechanical loads. 


\section{IMPURITY POWDER DROPPER APPARATUS}

The Impurity Powder Dropper as described in [10] has been installed on the AUG tokamak and is comprised of four powder feeders arranged at 90 degree intervals around a central aperture. Powder is fed to the individual IPD heads by means of funnel shaped reservoirs attached to cylindrical containers which provide additional inventory. The powder is advanced down the feed tray by means of an amplified sinusoidal voltage administered to the piezoelectric crystals below the feeder tray. Motion of the tray is monitored by an accelerometer mounted to the back of each individual assembly, and the signals are digitized. Crosstalk between the injectors is avoided by engineering distinct resonant frequencies for each slide tray, by adding small but different amounts of weight to each tray.

The IPD was installed in sector 14 on the top of AUG and connected to the main vessel through a $2.5 \mathrm{~m}$ long drop tube. An image of the installation as well as a cutaway drawing of the interior of the powder dropper assembly can be seen in figure 1. As the falling powder transits the vertical drop tube, it passes by a calibrated flow meter. In the flow meter, a fiber-fed LED source is used to generate a collimated beam which intersects the powder path and is then refocused into a return fiber connected to a photodiode. The greater the occultation provided by the transiting powder, the larger the attenuation, as can be seen in figure 2 . The bottom panel of the figure displays a typical excitation for the accelerometer attached to the powder dropper tray. After a ramp-up period of approximately $500 \mathrm{~ms}$, the accelerometer reaches a near constant signal voltage which continues for the duration of the powder injection. The upper panel in figure 2 shows the occultation of the flowmeter light level due to the falling powder. This period between the onset of the noise and the drop in light level caused by the falling powder represents the amount of time required for the powder to fall from the dropper assembly to the flowmeter location. Once the powder stream reaches the flowmeter location there is an observed deflection of the powder dropper flow meter signal in response to the transiting powder.This provides an estimate of the mass drop rate. This information and the duration of the drop allows an estimation of the total quantity of material deposited 
into the discharge. For the low-z compounds tested in AUG, drop rates ranging from 2 $\mathrm{mg} / \mathrm{s}$ to $200 \mathrm{mg} / \mathrm{s}$ have been reliably and repeatedly achieved in laboratory tests.

Testing of these powder dropper feeders has also determined that the length of the injection is limited only by the available quantity of powder. In addition the rapid response rate of the powder delivery and the ability to generate an arbitrary excitation pulse train means that this apparatus can be used multiple times in a discharge. This is especially noteworthy as the world fusion program begins to explore the complex physics of long pulse discharges.

The impurity powder dropper is unique in that is has the ability to drop many different impurity species utilizing the same delivery mechanism. The dropper has been tested with a range of impurities including boron, boron nitride, boron carbide, silicon carbide, elemental silicon, tin and lithium. For these first experiments, only two feeders were filled, the first with $5 \mu \mathrm{m}$ diameter BN powder, and the second with $70 \mu \mathrm{m}$ B powder; the other two assemblies were left empty as can be seen in the panel c) of figure 1 .

\section{BORON POWDER INJECTION EXPERIMENTS}

For this series of experiments, a set of first $\mathrm{BN}$ and then $\mathrm{B}$ containing discharges were interspersed with test target discharges designed to determine if there was a notable conditioning effect due to the injected materials[21]. Following this sequence of injection experiments was a set of RMP ELM suppression discharges to test access to low collisionality. Plasma conditions for the particulate injection shots were as follows: $I_{P}=$ $0.8 \mathrm{MA}, \mathrm{B}_{\mathrm{T}}=-2.5 \mathrm{~T}, \mathrm{P}_{\mathrm{NBI}}=10 \mathrm{MW}, \mathrm{P}_{\mathrm{EC}}=0.8 \mathrm{MW}$ with a density target of $\mathrm{n}_{\mathrm{e}}=6.5 \times 10^{19}$ $\mathrm{m}^{-3}$, with $\mathrm{I}_{\mathrm{P}}$ being a measure of the plasma current, $\mathrm{B}_{\mathrm{T}}$ being a measure of the toroidal field and $\mathrm{P}_{\mathrm{NBI}}$ and $\mathrm{P}_{\mathrm{EC}}$ as the quantity of neutral beam and electron cyclotron heating respectively. These conditions were chosen to generate robust ELM activity, thus providing 'good' contact between the SOL and the PFCs. It is believed that filamentary structures associated with the outward radial ELM transport are responsible for both 
initial boron distribution as well as sputtering and redistribution of deposited boron content, thus providing a net material transport to the divertor as the discharge progresses.

The equilibrium shape utilized for the conditioning discharges can be seen in panel a of figure 3 where the blue arrow denotes the location of the powder dropper tube in relation to the conformal ASDEX discharge. The drop tube is located in a position near the upper extent of a discharge in lower-single null configuration (LSN), with a radial location 23 $\mathrm{cm}$ outboard of the geometric plasma center, thus the initial plasma material interactions occurs towards the low field side of the discharge before being entrained within the plasma flow as described in [21]. This location is further clarified by the image of the interior of the vacuum vessel in panel 2 where the drop tube aperture is highlighted by the blue circle. The introduction of boron-based powders was performed at injection levels up to $60 \mathrm{mg} / \mathrm{s}$ for 3 seconds during the $\mathrm{H}$-mode flat top at constant injection rates. True color images of plasma operations are shown in panels c) and d), which display active boron nitride and boron powder injection respectively. The bright blue emission observed here is believed to be due to singly ionized boron at $412.2 \mathrm{~nm}$, with NII emission at $399.5 \mathrm{~nm}$ contributing to the signal for BN injections. These wide-angle images showed that the injected particles rapidly ablated upon contact with the boundary plasma, consistent with observations of $\mathrm{B}_{4} \mathrm{C}$ granule ablation in DIII-D[22]. There was also an observed tendency for B powder to agglomerate into larger clumps, thus leading to a pulsed, intermittent boron injection. These boron clusters provided concentrated particle sources allowing deeper penetration into the discharge, due to the self-shielding of the larger particles as observable from the formation of ionized field-aligned emissions within the discharge.

The quantities of boron injected during the series of discharges are summarized in table 1 . As a fraction of the total inventory, $88 \%$ of the total injected boron was introduced in the final two discharges, with $60 \%$ being deposited in the final conditioning discharge. These quantities were tabulated by the flow meter and serve as an upper limit of the amount injected into the plasma. Experiments with a similar powder dropper apparatus on DIII-D[23] have shown the accumulation of powder around the drop tube location, 
possibly due to electrostatic charging of the dropped powders. Further characterization of the injection efficiency is ongoing.

\begin{tabular}{|l|l|l|l|}
\hline $\begin{array}{l}\text { AUG } \\
\text { Discharge }\end{array}$ & $\begin{array}{l}\text { Injection } \\
\text { Species }\end{array}$ & $\begin{array}{l}\text { Quantity of boron } \\
\text { injected (atoms) }\end{array}$ & $\begin{array}{l}\text { Cumulative } \\
\text { boron injection }\end{array}$ \\
\hline 34796 & BN & $1.7 \times 10^{19}$ & $1.7 \times 10^{19}$ \\
\hline 34798 & BN & $1.7 \times 10^{20}$ & $1.9 \times 10^{20}$ \\
\hline 34799 & BN & $3.5 \times 10^{20}$ & $5.4 \times 10^{20}$ \\
\hline 34801 & BN & $1.6 \times 10^{21}$ & $2.2 \times 10^{21}$ \\
\hline 34803 & BN & $1.4 \times 10^{21}$ & $3.6 \times 10^{21}$ \\
\hline 34808 & B & $9.6 \times 10^{20}$ & $4.5 \times 10^{21}$ \\
\hline 34809 & B & $3.6 \times 10^{21}$ & $8.1 \times 10^{21}$ \\
\hline 34825 & B & $1.0 \times 10^{22}$ & $1.9 \times 10^{22}$ \\
\hline
\end{tabular}

Table 1: List of IPD boron powder injection discharges with injected quantities and cumulative boron deposition

\section{1: BORON NITRIDE POWDER INJECTION}

Boron nitride was chosen for the preliminary impurity injection discharges due to the enhanced delivery accuracy of the IPD with this material at lower injection levels, thus allowing a smooth initial introduction of powder. In this series of five BN conditioning discharges, injected $\mathrm{B}$ was varied from $\sim 1.7 \times 10^{19}$ atoms/discharge to $\sim 1.6 \times 10^{21}$ atoms/discharge. Test discharges were interspersed with the conditioning discharges to assess the impact and transport of the injected powder, and to classify the plasma response. The highest injection rate was limited by the concomitant introduction of radiative nitrogen into the discharge, with an observed increase in radiated power $\left(\mathrm{P}_{\mathrm{RAD}}\right)$ by a factor of 1.8. Globally the BN injection improved energy confinement by $10-20 \%$, levels similar to those previously attributed to an improvement in pedestal stability during nitrogen gas injection. 
Initial questions about the assimilation efficiencies into the discharge were answered by time resolved line emission intensities as measured by visible spectroscopy at the ICRH limiter and the divertor. Both locations showed increases in the boron and nitrogen signal levels coincident with the period of powder introduction, as can be seen in figure 4 . Strong increases in the impurity signatures are observed shortly after the introduction of material and increased signal levels are evident for the remainder of the injection period. These measurements suggest that the injected powder is being transported to the surfaces of enhanced plasma material interaction, leading to conditions which should be favorable to deposition and resultant coating. This appears to be a relevant difference between the application of plasma assisted deposition coatings and those applied through glow discharge or evaporation. For the IPD method of material deposition, the primary locations to which material is applied are those with the strongest plasma contact i.e. the limiter and divertor surfaces.

The relative efficiencies with which the material was assimilated into the discharge can be determined by comparing relative nitrogen signal intensities. In figure 5 the traces represent the N6+ intensities recorded at the radial coordinate $\rho_{\phi} \sim 0.7$ for both the BN containing and the conditioning test discharges. For the lowest level of injection (AUG \#34726) the injection amount of $1.7 \times 10^{19}$ atoms is not resolvable above the background level, which is displayed by the thin black lines. For the next two discharges (AUG \#34798 \& \#34799) injection levels of 1.7 and $3.5 \times 10^{20}$ atoms provide nitrogen signal intensities substantially above the background level. Injection quantities increased by an additional factor of 5 for discharges AUG 34801 and 34802 show an increase in peak signal intensity by nearly a commensurate amount. By comparing these traces with the tabulated values we find that the relative signal intensities as shown by the colored traces roughly correspond to the injected quantities of material as determined by the flowmeter calibration. We also find that there is minimal inter-shot retention of nitrogen, as evidenced by the overlaid black traces corresponding to the series of conditioning test discharges. 
While not the primary objective of the wall conditioning study, the introduction of nitrogen-containing powders and subsequent incorporation into the discharge had a notable effect on overall discharge evolution. It has been observed on devices with metal walls such as JET-ILW (ITER Like Wall) and AUG that the introduction of low Z impurities (such as nitrogen or deuterated methane (CD4)) provides increased divertor radiation and improved energy confinement improvement, partially bridging the gap between metal wall and carbon wall observed energy confinement levels[24]. These and other low-Z impurities have radiation profiles which peak below $100 \mathrm{eV}$, allowing enhanced edge radiation to cool the near-separatrix and scrape-off layer, but become fully stripped once integrated into the core discharge avoiding deterioration of the pedestal. Confinement and total stored energy improvement observed with $\mathrm{N}_{2}$ seeding on AUG is on the order of $20-30 \%$ [25], primarily due to an increase in ion and electron pedestal temperature and a core temperature profile stiffness which increases plasma pressure. The elevated $\beta_{\mathrm{N}}$ has a subsequent effect on pedestal ballooning stability, which enhances the pedestal pressure gradients. The observation of a degradation in the High Field Side High Density (HFSHD) region[26, 27] of the SOL by approximately $50 \%$ with increasing $\mathrm{N}$ radiation has been found to correlate with pedestal confinement improvements[28]. It is thought that a reduction in the density of the HFSHD region reduces the plasma fueling source and allows the density profile to shift inward, improving peeling-ballooning stability. Note that the pedestal top densities and ELM frequencies observed in nitrogen seeded discharges are relatively unaffected by seeding.

During the IPD injection experiments recently performed on AUG, for boron nitride injection rates of $12 \mathrm{mg} / \mathrm{s}, \mathrm{n}_{\mathrm{e}}, \mathrm{P}_{\mathrm{RAD}}$ and $\mathrm{W}_{\mathrm{MHD}}$ were all seen to increase from the start of the injection until $\mathrm{t}=5.5 \mathrm{~s}$ at which point the discharge became MHD unstable, as can be seen in figure 6 . The upper panel of the figure shows radiated power increasing by nearly $50 \%$ from 3.3 MW to $6 \mathrm{MW}$ with the middle panel showing an edge density increase of $25 \%$. The lower panel shows enhancements to the $\mathrm{H}_{98}$ confinement factor and a $20 \%$ increase in $\mathrm{W}_{\mathrm{MHD}}$ from 0.55 to $0.66 \mathrm{MJ}$. At $5.5 \mathrm{~s}$ the resulting increase in performance is such that $\beta_{\mathrm{N}}$ reaches 2.7 , which is the typical neoclassical tearing mode threshold; indeed an MHD instability and subsequent loss of performance are observed. These 
improvements in performance are similar to those observed at AUG[29] and JET-ILW[30] when a nitrogen gas puff is introduced.

To better classify the increase in performance, as shown in figure 7 , we compare the averaged electron density and temperature profiles for a BN conditioning discharge with a discharge with the same conformal shape and heating of a conditioning discharge, but without the $\mathrm{BN}$ injection. It can be seen that the enhanced performance during $\mathrm{BN}$ injection compared with the other two discharges is solely observed within improvements to the density pedestal. This is in contrast to gaseous nitrogen seeded discharges[20] where pedestal densities were unaffected by the injection while there was noted improvement to the pedestal temperature. To expand this comparison we plot in figure 8 the evolution of the full profiles for discharges with BN injection (AUG 34801), B injection (AUG 34825), and no injection (AUG 34802) during the period before injection, just at the start of injection and after a full second of injection. Again we note the $20 \%$ increase in pedestal density coincident with $\mathrm{BN}$ injection which is not observed in the other two cases.

The presence of elevated nitrogen emission from the divertor indicates the ability of injected $\mathrm{BN}$ to also enhance the radiation in the divertor. The tomographic reconstructions in figure 9 compare the divertor radiated bolometry profiles of the control test discharge AUG 34802, and a discharge containing BN injection (AUG 34801) with a standard gaseous nitrogen seeded discharge (AUG 31228) included for comparison. The addition of $\mathrm{BN}$ increases the total radiated power by approximately $50 \%$, which in turn increases the radiated fraction of the $10.8 \mathrm{MW}$ input power $\left(\mathrm{P}_{\mathrm{NBI}}=10 \mathrm{MW}, \mathrm{P}_{\mathrm{ECRH}}=0.8\right.$ $\mathrm{MW}, \mathrm{P}_{\mathrm{ICRH}}=0.0 \mathrm{MW}$ ) from $54 \%$ for the unseeded discharge to almost $80 \%$. Figure. $9 \mathrm{a}$ shows the profile from a standard nitrogen seeded discharge (AUG31228) with $\mathrm{P}_{\mathrm{TOT}}=$ 13.6 MW $\left(\mathrm{P}_{\mathrm{NBI}}=10 \mathrm{MW}, \mathrm{P}_{\mathrm{ECRH}}=1.7 \mathrm{MW}, \mathrm{P}_{\mathrm{ICRH}}=1.8 \mathrm{MW}\right)$ : we can see that the radiated power fraction $\sim 81 \%$ is equivalent to that during $\mathrm{BN}$ injection. There is variation in the location of the radiated power as the concentration of gaseous seeded radiation appears to be located near the so-called High Field Side High Density (HFSHD) 
[26] region while radiation enhancement from the $\mathrm{BN}$ injected discharge is observed as an augmentation of the preexisting radiation pattern.

Given that $\mathrm{BN}$ and $\mathrm{N}_{2}$ provide similar confinement enhancements to their respective reference discharges, it is instructive to compare the relative efficiencies of the injected materials in providing those benefits. Comparing the signatures from the residual gas analyzer installed on ASDEX Upgrade[31, 32], we can begin to examine the relative quantities of nitrogen introduced into the discharge as well as the quantity of ammonia produced by that injection. Figure 10 shows that both the level of nitrogen observed and the level of ammonia produced is at least an order of magnitude less for the IPD injection discharges than for the gas seeded discharges[33]. This can be simply understood by examining the quantity of material introduced during the respective discharges. During the gas puff discharge (AUG 31228) $4.2 \times 10^{22}$ nitrogen atoms were injected into the vessel while the number of nitrogen atoms introduced by the powder dropper for the respective discharge (AUG 34801) was $1.4 \times 10^{21}$ atoms. Thus it appears that the introduction of $\mathrm{BN}$ is possibly a more efficient manner of recovering pedestal performance for a metal wall machine. This being said, the results presented here are preliminary and the RGA only monitors a single gas loss channel, as such further study is needed to determine the overall applicability of these results.

The quantity of ammonia produced by these two methods is particularly relevant for future devices: an elevated presence of tritiated ammonia in ITER would both reduce the available tritium inventory[34], and introduce a chemically reactive substance that can shorten component lifetime in the tritium reprocessing plant. However, we note as it pertains to tritium retention levels that previous studies of fuel retention with carbon[35, 36] show elevated fuel retention levels. As such, the intoroduction of boron, with a similar affinity to carbon, may result in further fuel retention due to co-deposition of tritiated borane. In addition the possible accumulation of tritiated dust in remote areas within the vacuum vessel could result in the need for more frequent dust mediation. As such, careful consideration would need to be given prior to the injection of boron containing powders into active discharges on JET-ILW or ITER. 
As it pertains to the primary thrust of this experiment, $\mathrm{BN}$ was not deemed to be an effective injection material to provide wall conditioning as $\mathrm{B}$ and $\mathrm{N}$ atoms are injected in equal amounts and the effects are dominated by the strongly radiative nitrogen. Thus, to continue the conditioning study a change from $\mathrm{BN}$ to pure $\mathrm{B}$ injection was then undertaken as the pure B allowed a larger material introduction without the subsequent (albeit positive) effect on discharge performance.

\subsection{BORON POWDER INJECTION}

Following the $\mathrm{BN}$ injection experiment, three discharges with increasing $\mathrm{B}$ injection rates were performed. The conditioning discharges were found to be compatible with large quantities of boron. Injection rates of up to $60 \mathrm{mg} / \mathrm{s}$ corresponding to $9.2 \times 10^{21}$ atoms over the course of the discharge resulted in minimal impact on plasma performance. As is shown in figure 11 the deposition of boron powder resulted in only a $5 \%$ increase in electron density and up to a 50\% increase in radiated power. In addition, there was no strong effect on plasma stored energy or change in confinement due to the injection of atomic boron.

The core concentration of $\mathrm{B}$ was measured by charge exchange recombination spectroscopy (CXRS). Figure 12 provides multiple snapshots of boron concentration over the course of the discharge. The emission data are analyzed by the charge exchange concentration analysis code (CHICA)[37]. Within the CHICA code, reconstruction of accurate impurity profiles across multiple diagnostic chords are provided through a forward model of the edge impurity densities utilizing plasma equilibrium and kinetic profiles. Boron injection begins at $4 \mathrm{~s}$, thus the first panel displays the baseline boron concentration prior to active injection. In the remaining time slices the concentration of boron is seen to increase with core densities raising from $5 \times 10^{17} \mathrm{~m}^{-3}$ after approximately $1 \mathrm{~s}$ of injection, to an average of $2.5 \times 10^{18} \mathrm{~m}^{-3}$ after nearly the full $4 \mathrm{~s}$ of injection. Also of interest is the rate of reduction in B concentration after active introduction is stopped. 
The concentration of boron drops by a factor of 2.5 over the course of $200 \mathrm{~ms}$, indicating a rapid outward transport of material, presumably due to the $\sim 100 \mathrm{~Hz}$ ELM frequency.

Examining the full radial profile of boron density and concentration in figure 13 we observe the B5+ density profiles evolve from hollow to slightly peaked with the concentration remaining flat for the majority of the discharge. The strong increase in boron signal at $\mathrm{t}=7 \mathrm{~s}$ is coincident with the stepwise reduction of neutral beam power from $10 \mathrm{MW}$ to $5 \mathrm{MW}$ and a commensurate reduction in the ELM rate from greater than 100 $\mathrm{Hz}$ to $50 \mathrm{~Hz}$. Examining panels d) and e) which shows the electron density and fractional boron concentration respectively we see that even with the increased B confinement at the end of the discharge boron concentration does not increase past $6 \%$. While this is substantially larger than the N7+ and C6+ concentrations of $1.5-2 \%$ seen with gaseous nitrogen or methane injections[28], there were no obvious indications of instabilities that would suggest a limit for material injection into these types of discharges.

\section{OBSERVATIONS OF CONDITIONING EFFECTS}

Reference shots to evaluate the wall conditioning effects following B injection were interleaved with the powder deposition discharges (both $\mathrm{BN}$ and $\mathrm{B}$ ) and these results are discussed in Ref.[21] and summarized here. For the reference shot chosen, $\mathrm{D}_{2}$ fueling was terminated at $\mathrm{t}=3.5 \mathrm{~s}$, with core tungsten accumulation leading to an unstable discharge evolution observed without IPD B injection. This scenario was undertaken with the assumption that these unstable discharges would perform better if the wall conditions were improved with the accumulated B coatings, even if no B were injected directly into these discharges. In discharge \#34797, undertaken at the start of the powder injection program, there was an observed strong MHD event at $\mathrm{t}=5.8 \mathrm{~s}$ followed by a radiatively induced disruptive event at $t=8 \mathrm{~s}$. This is contrasted with discharge \#34810 which was undertaken after cumulative injection of $5.8 \times 10^{21}$ boron atoms. This discharge was successful in reaching the $I_{P}$ ramp down at $t=9 \mathrm{~s}$ with reduced levels of radiative losses albeit while still exhibiting some unstable behavior. A pair of spectroscopic cameras entitled LVL and DVL respectively monitor impurity influx at the ICRF limiter and the 
divertor respectively. They monitor, among other things, the line emission from OII, BI and WI. Observations of the time averaged brightness of the BII and OII emission at the limiter during the $\mathrm{H}$-mode flat top shows an increase in the former by $25 \%$ and a decrease in the latter by $30 \%$ over the course of the injection discharge series as can be seen by figure (8) of Ref [21]. These observations are consistent with the newly deposited boron substrate gettering the available oxygen, from e.g. classic boronization.

To further test the conditioning effect of the injected B powder, a sequence of discharges with resonant magnetic perturbations (RMP) for ELM suppression were conducted immediately after the final discharge of the conditioning campaign. Due to the operational scenarios, these RMP discharges are very sensitive to wall conditions[38, 39], and thus are only attempted within a few days of a standard boronization. A portion of this sensitivity has been found[18] to be due to the tungsten limiters which protect two of the ICRH antennas. Sputtering from this limiter provides a tungsten source which can lead to radiative collapse of the discharge, therefor a robust boron coating over this limiter helps maintain radiated power at an acceptable level during ELM-free operation. These experiments were conducted at the end of a boronization cycle and as such the wall conditions, while tolerable for standard plasma operation, were believed to be too poor to achieve required low collisionality regime necessary for RMP ELM suppression.

As is shown in figure 14, following the B conditioning discharges all three attempts to run low density discharges with ELMs suppressed by magnetic perturbations were successful. For the three equivalent discharges there was no evidence of runaway radiation levels, there was a strong density pump out, and full mitigation of the ELMs. We were not able to continue the shot series to determine when the coating was completely worn away and the conditioning effect diminished, however the slightly elevated $\mathrm{P}_{\mathrm{RAD}}$ seen for the blue trace representing AUG \#34829 seen in the bottom panel of figure 14 may indicate that we were starting to wear away the coating. Given the success of these attempts it is then reasonable to compare the RMP ELM suppression discharge which was achieved through particulate injection with one which is accessed through standard boronization methods. Figure 15 shows an overlay of two discharges, 
one (AUG \#34829) attempted after particulate boron injections as discussed in this paper, and one (AUG \#34214) which was conducted 2 days (25 discharges) after a standard boronization. As can be seen, both discharges exhibit similar levels of wall conditioning early in the discharge evolution as evidenced by the density pump out which allows full ELM suppression and a maintained level of low radiated power. The deviation between the evolution of the discharges is believed to be due to a nitrogen gas puff introduced at $\mathrm{t}$ $=4.5 \mathrm{~s}$ into the powder conditioned discharge which not present in the comparison discharge.

Unfortunately, a pre-IPD ELM suppression attempt with RMP was not undertaken. Thus the positive impact of the $\mathrm{B}$ conditioning on access to low collisionality discharges remains suggestive but not fully conclusive until the experiment is repeated with a control discharge. Moreover while these results are consistent with the deposition and coating of the relevant surfaces with an effective boron layer, it must be noted that there were no direct measurements made of the wall composition or coating thickness during these experiments - that also is planned in the future.

\section{SUMMARY AND CONCLUSION}

Solid impurity powders have been injected into ASDEX Upgrade to explore the possibility of conditioning by means of particulate injection. Boron nitride and boron powders have been injected at rates up to $50 \mathrm{mg} / \mathrm{s}$ and $75 \mathrm{mg} / \mathrm{s}$ respectively into conformal LSN ELM-y H-mode discharges with the shaping and ELM frequency chosen to maximize contact between the discharge and the main chamber walls. Boron nitride injection into plasmas increased the core energy confinement by $10-20 \%$, qualitatively similar to nitrogen gas injection. However, the $\mathrm{BN}$ injection appeared to increase pedestal density, while nitrogen gas injection increased pedestal temperature. On the other hand, the amount of ammonia production was reduced by $>90 \%$ with solid powder injection. We note that the effect of $\mathrm{BN}$ injection into $\mathrm{H}$-mode plasmas is dominated by the nitrogen radiation, which sets a relatively modest upper limit on the powder injection rate. 
Solid B injection was also demonstrated. B injection appeared to improve wall conditions and discharge performance in reference discharges that were interleaved with conditioning discharges. Cumulative boron injection amounts greater than $100 \mathrm{mg}$ were observed to mildly reduce spectroscopic signals from both oxygen and tungsten at the limiter and divertor surfaces indicating an enhanced boron coating which getters the former and covers over the latter. However, we note that as there were no depositional diagnostics available at the time of the experiment a full understanding of the location and thickness of deposited coatings remains to be determined

As a fraction of the total inventory, $88 \%$ of the total $340 \mathrm{mg}$ of injected boron was introduced in the final two discharges, with $60 \%$ being deposited in the final conditioning discharge. Thus the observed good wall conditions are likely due to the final one or two conditioning discharges, as opposed to cumulative conditioning from the full sequence of nine $\mathrm{B}$ and $\mathrm{BN}$ discharges. In addition, observations of the shot evolution show no indications that the final conditioning discharge was adversely effected by the injection and as such there remains the possibility to increase the injection rates for a more effective utilization of discharge time. In addition, low density discharges with magnetic perturbations and suppressed ELMs were successfully accessed after the B conditioning discharges; historically such discharges are accessible only after a recent conventional boronization.

As the deposition of the material is localized to areas of active plasma activity, this method of coating replenishment is expected to be especially effective in extending the lifetime of deposited boron layers on areas prone to rapid erosion. These areas include plasma strike points and limiting surfaces especially where application of the ICRF antennas are concerned. These preliminary results suggest that the application of BN can be used to both improve plasma performance in real-time, and that the introduction of pure B can lead to improved wall conditions as manifest by improved plasma performance in subsequent RMP ELM suppression tests. Furthermore as the injection system is capable of injecting a wide number of impurities $\left(\mathrm{B}, \mathrm{BN}, \mathrm{B}_{4} \mathrm{C}, \mathrm{Li}, \mathrm{C}, \mathrm{Sn}, \mathrm{Mo}\right.$, $\mathrm{W}, \ldots)$ it can be utilized for a range of studies. In addition, a delivery device such as the 
IPD could be envisioned as a method for intra-shot conditioning to allow extreme long pulse discharges. Such conditioning could be required due to deterioration of low $\mathrm{Z}$ coatings on metal walls and the resultant increase in high $\mathrm{Z}$ sputtering. Future experiments will focus on measuring the conditions of the wall surfaces before, during and after powder injection, and proving that the powder conditioning is unequivocally responsible for opening access to low density, ELM suppressed discharges far from a boronization.

Future research utilizing this technique will evaluate of the efficacy of coating by utilizing active plasma deposition when compared to other proven techniques such as coating by RF plasmas. In addition future modelling efforts will be focused on determining with better certainty the active deposition locations and amounts deposited as a fraction of injected material. Even so, the utilization of a powder dropper based real time conditioning technique holds promise for potential future applications and warrants further study especially as it concerns its applicability to long pulse devices.

\section{ACKNOWLEDGEMENTS}

This work has been carried out within the framework of the EUROfusion Consortium and has received funding from the Euratom research and training programme 2014-2018 and 2019-2020 under grant agreement number 633053. The views and opinions expressed herein do not necessarily reflect those of the European Commission. The U.S. authors are supported in part by U.S. Dept. of Energy under contract DE-AC02-09CH11466.

\section{DISCLAIMER}


This report was prepared as an account of work sponsored by an agency of the United States Government. Neither the United States Government nor any agency thereof, nor any of their employees, makes any warranty, express or implied, or assumes any legal liability or responsibility for the accuracy, completeness, or usefulness of any information, apparatus, product, or process disclosed, or represents that its use would not infringe privately owned rights. Reference herein to any specific commercial product, process, or service by trade name, trademark, manufacturer, or otherwise, does not necessarily constitute or imply its endorsement, recommendation, or favoring by the United States Government or any agency thereof. The views and opinions of authors expressed herein do not necessarily state or reflect those of the United States Government or any agency thereof. 
[1] Winter J., 1996 Plasma Phys. Control. Fusion 381503

[2] ITER Physics Expert Group on Disruptions, Plasma Control and MHD et al. 1999 Nucl. Fusion 392577

[3] Douai D. et al., 2013 J. Nucl Mater. 438 S1172

[4] Kugel H. et al., 2003 J. Nucl. Mater. 313 (2003) 187

[5] Rohde V. et al., 2007 J. Nucl. Mater. 363-365 1369

[6] Jackson G.L et al., 1991 Phys. Rev. Lett 673098

[7] Hu J.S. et al., 2014 Fusion Eng. Des. 892875

[8] Maingi R. et al., 2012 Nucl. Fusion 52083001

[9] Osborne T et al., 2015 Nucl. Fusion 55060018

[10] Nagy A. et al., 2018 Rev. Sci. Instr. 89 10K121

[11] Kallenbach A. for the ASDEX Upgrade Team and the EUROfusion MST1 Team 2017 Nucl. Fusion 57102015

[12] Neu R. et al., 2013 J. Nucl. Mater. 438 S34-S41

[13] Herrmann A. et al., 2015 Nucl. Fusion 55063015

[14] Dux R. et al., 2009 J. Nucl. Mater. 390-391 858

[15] Rohde V. et al,. 1999 ECA Proceedings of the 26th EPS Conference on Controlled Fusion and Plasma Physics 23J 1513

[16] Suttrop W. et al., 2017 Plasma Phys. Control. Fusion 59014049

[17] Bobkov V. et al., 2010 Nucl. Fusion 50035004

[18] Dux R. et al., 2007 J. Nucl. Mater. 363-365 112

[19] Kallenbach A et al., 2015 Nucl. Fusion 55053026

[20] Schweinzer J. et al., 2011 Nucl. Fusion 51113003

[21] Bortolon A. et al., 2019 Nucl. Mater. Energy 19 (2019) 384

[22] R. Lunsford et al., 2019 Nucl. Mater. Energy 19 (2019) 34

[23] Bortolon A. et al., 2018 Bull. Am. Phys. Soc. 63

http://meetings.aps.org/Meeting/DPP18/Session/UP11.41 
[24] Beurskens M.N.A. et al., 2013 Plasma Phys. Control. Fusion 55124043

[25] Kallenbach A. et al., 2013 Plasma Phys. Control. Fusion 55124041

[26] Potzel S. et al., 2015 J. Nucl. Mater. 463541

[27] Potzel S. et al., 2014 Plasma Phys. Control. Fusion 56025010

[28] Giroud C. et al., 2013 Nucl. Fusion 53113025

[29] Beurskens M.N.A. et al., 2016 Nucl. Fusion 56056014

[30] Dunne M.G. 2017 Nucl. Fusion 57025002

[31] Neuwirth D. et al., 2012 Plasma Phys. Control. Fusion 54085008

[32] Rohde V. et al., 2015 J. Nucl. Mater. 463672

[33] Drenik A. et al., 2019 Nucl. Fusion 59046010

[34] Laguardia L. et al., 2017 Nucl. Mater. Energy 12 261-266

[35] Brezinsek S. et al., 2013 Nucl. Fusion 53083023

[36] Doerner R.P. et al., 2009 Nucl. Fusion 49035002

[37] McDermott R. M. et al., 2018 Plasma Phys. Control. Fusion 60095007

[38] Suttrop W. et al., 2018 Nucl. Fusion 58096031

[39] Leuthold N. et al., 2017 Plasma Phys. Control. Fusion 59055004 


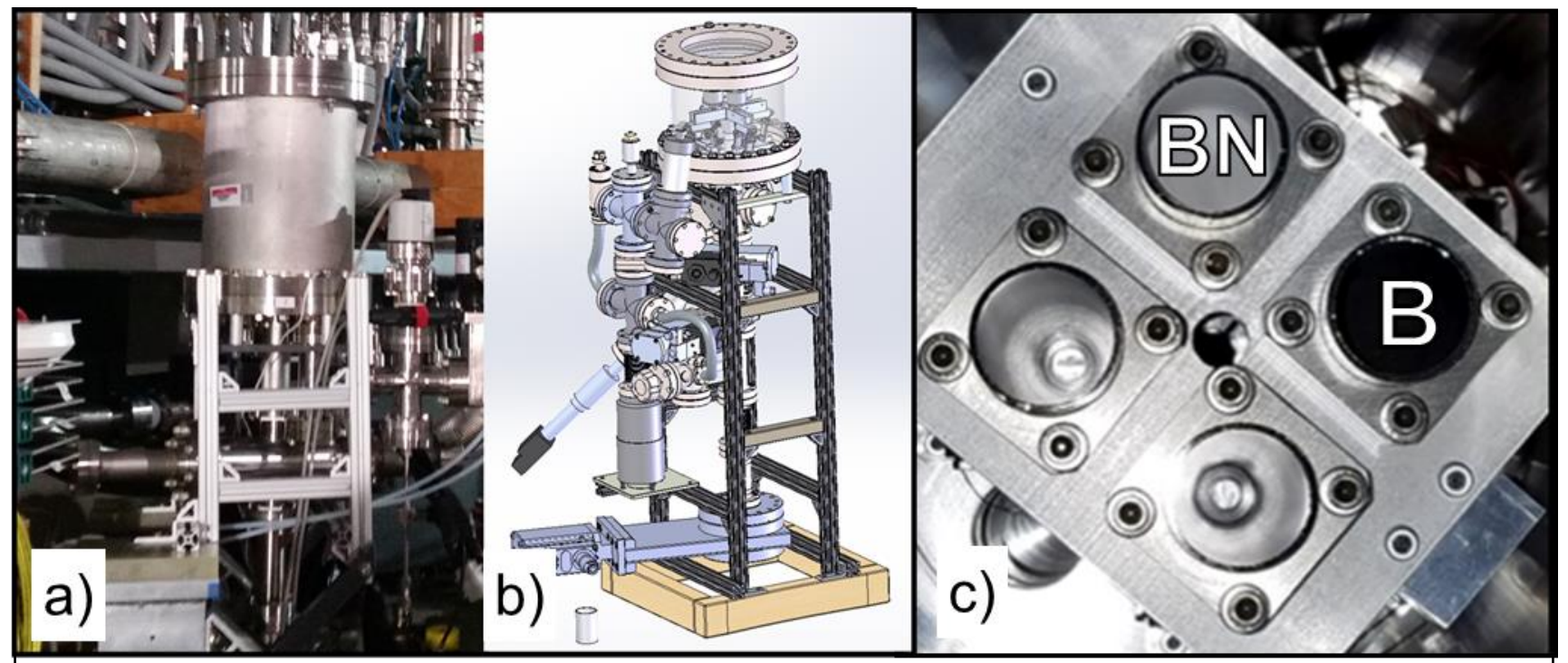

Figure 1: Impurity Powder Dropper (IPD): Panel a) shows an image of the IPD as installed on ASDEX-Upgrade. Panel b) shows a full $3 \mathrm{~d}$ model of the powder dropper systemincluding a cutaway of the dropper head. Panel c) is a top down view of the dropper reservoirs where two dropper heads have $\mathrm{BN}$ and $\mathrm{B}$ powders loaded while two heads remain empty. 


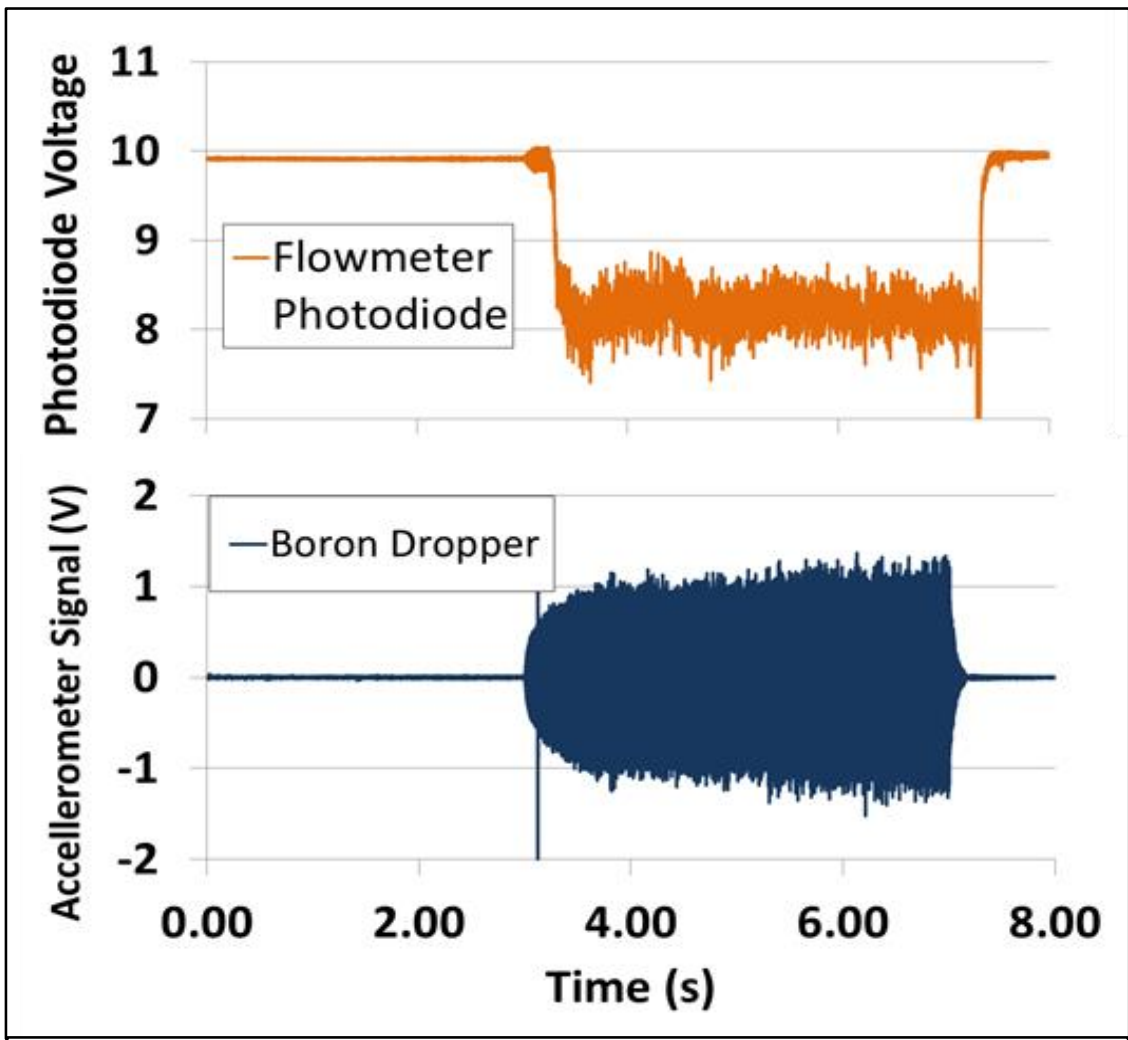

Figure 2: Exemplar traces from the IPD onboard diagnostics. The bottom figure displays an accelerometer trace from an active powder dropper apparatus. The piezoelectrics are excited from $3 \mathrm{~s}$ to $7 \mathrm{~s}$ with a pronounced ramp up but limited post-excitation decay. The upper figure displays the fractional occultation of the flowmeter photodiode by the falling powder. The $100 \mathrm{~ms}$ lag in response between the lower and upper graphs is due to the time required for the powder to drop from the feed tray to the photodiode location. The photodiode deflection noted here corresponds to an approximately $100 \mathrm{mg} / \mathrm{s}$ powder drop rate. 


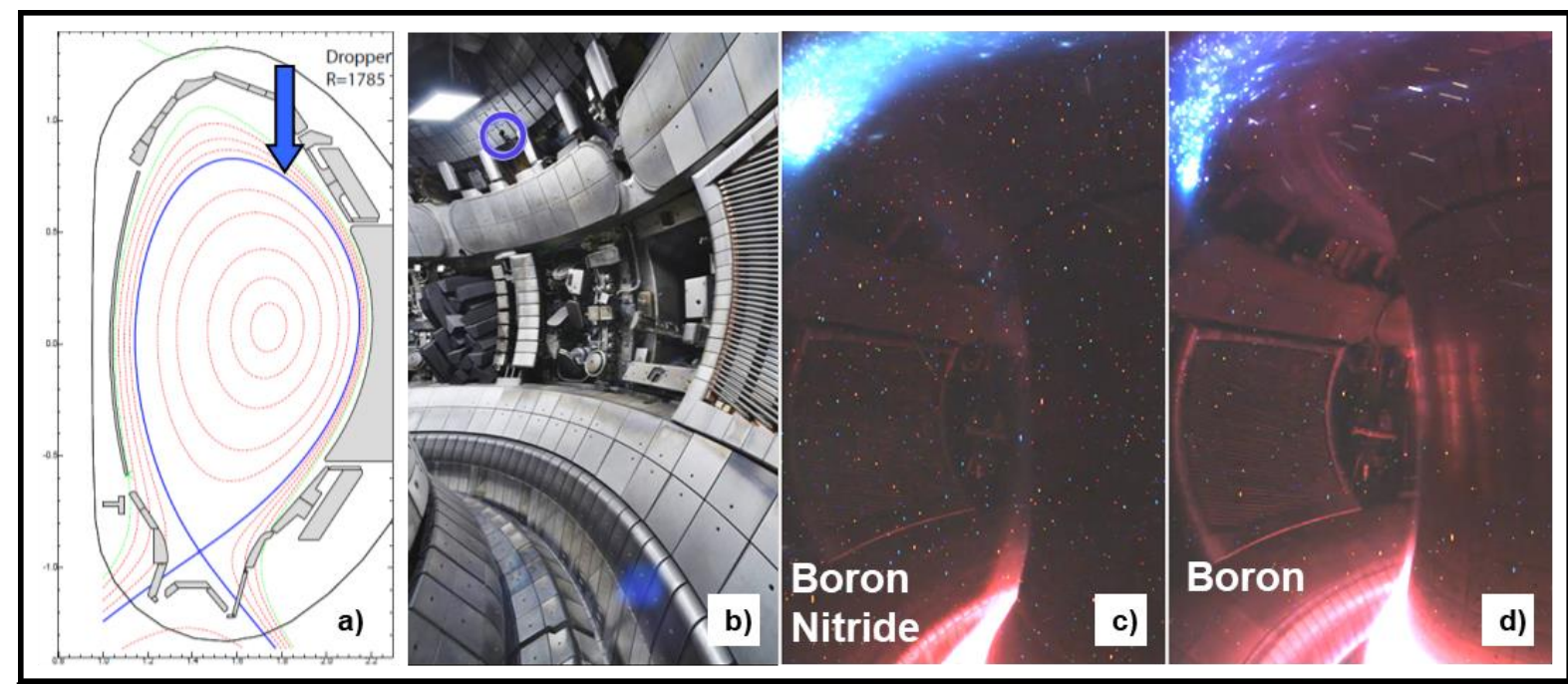

Figure 3: Powder dropper injection site : Panel a) displays the location of powder injection (blue arrow) with respect to the poloidal cross section of the conformal discharge reconstruction. The blue circle in Panel b) displays the location of the entry tube within the vacuum vessel. The blue shadow in the divertor in panel b) is an image artifact. Panels c) \& d) show active injection of BN and B respectively as recorded by a wide angle true color camera. 


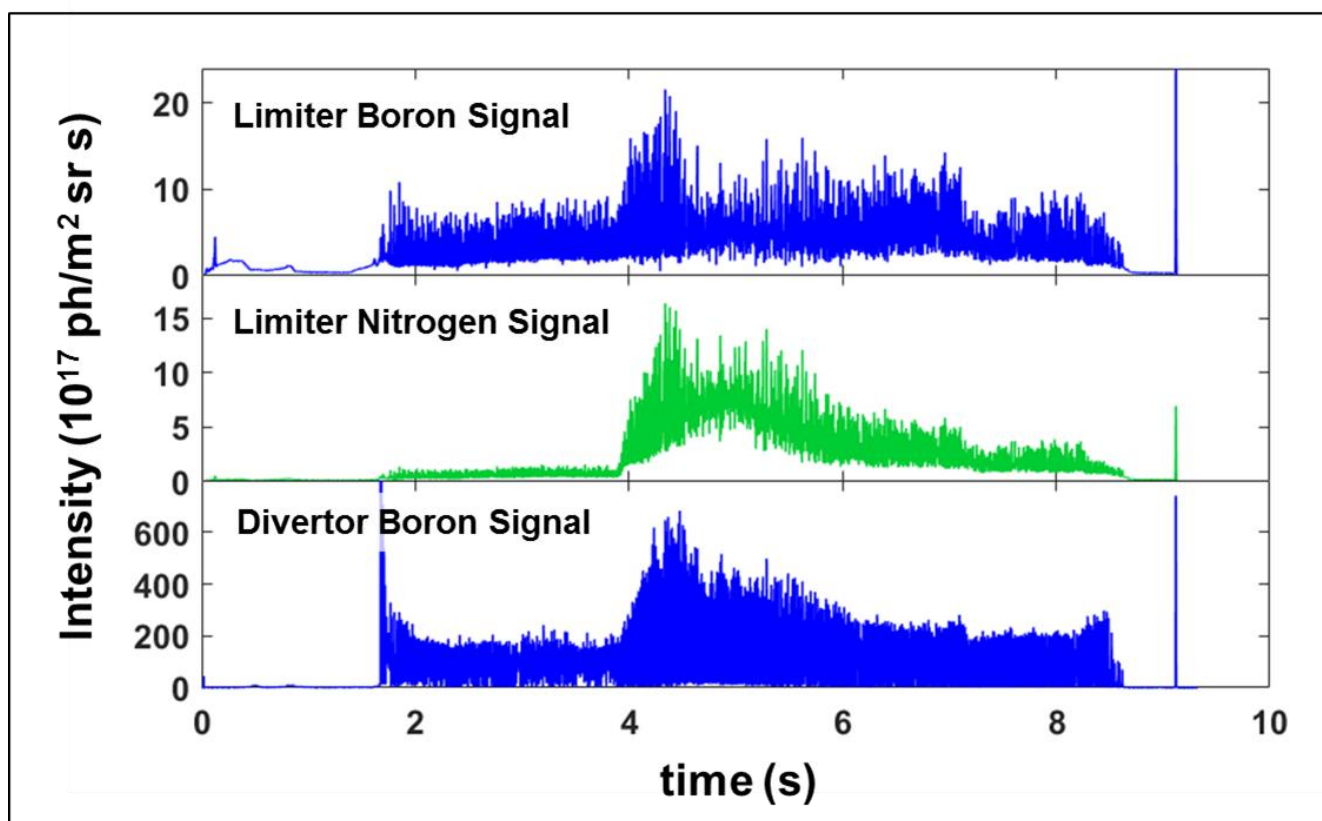

Figure 4 : Impurity signatures at the ICRH limiter and divertor surfaces as a result of boron nitride injection during AUG 34803. Powder injection starts at approximately $3.5 \mathrm{~s}$ and spectroscopic signatures show a strong impurity presence at $\mathrm{t}=4 \mathrm{~s}$ with a ramping increase in signal level for the next half second and an elevated signal level for the remaining injection period. 


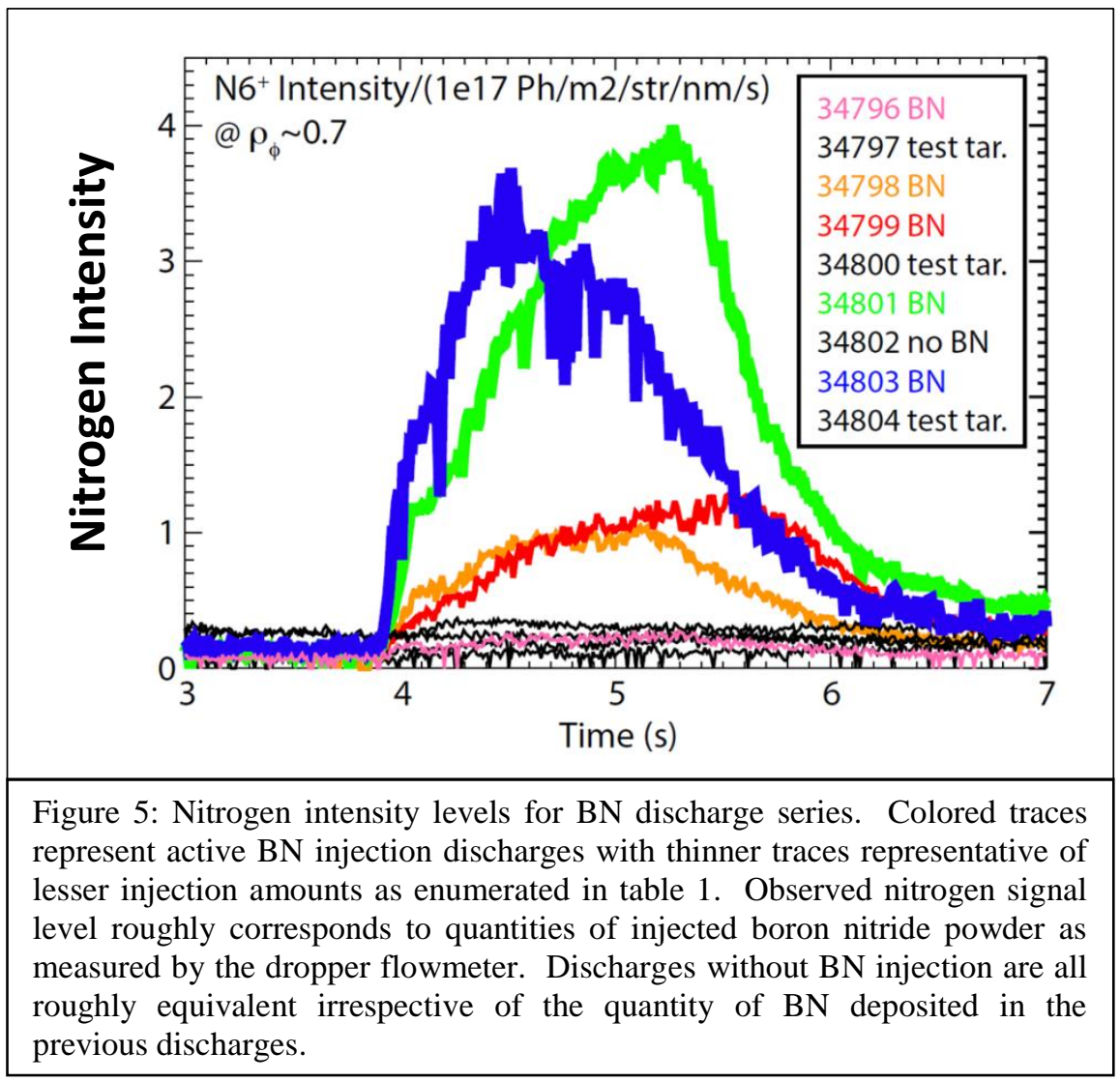



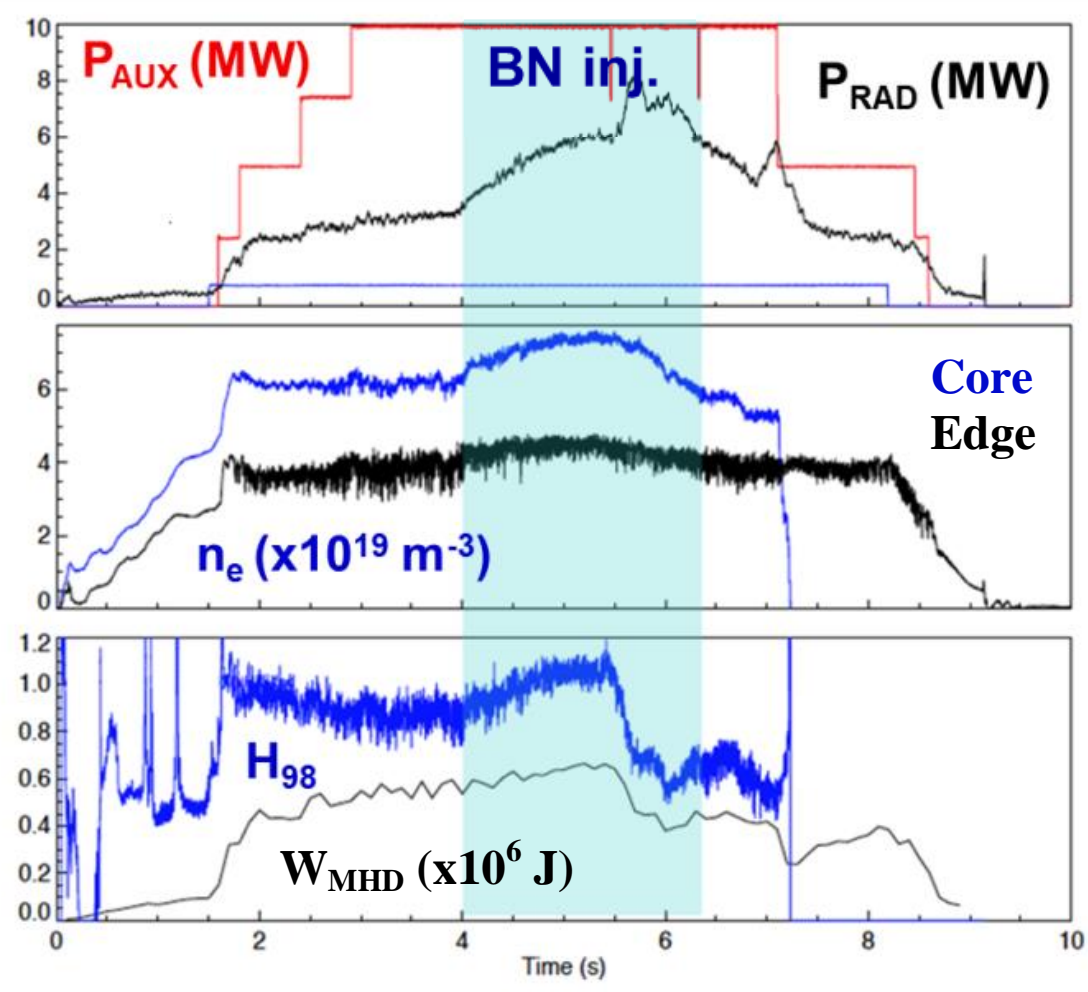

Figure 6: Effects of boron nitride injection on overall plasma parameters for discharge \#34801. The introduction of boron nitride powder to the edge of the discharge has a strong effect on overall plasma behavior. Due primarily to the simultaneous introduction of nitrogen with the boron there are increases in the radiated power (top panel) and core density (middle panel). In addition there are also observable increases in the stored energy and confinement (lower panel) prior to the observation of a locked mode at $t=5.3 \mathrm{~s}$. Period of injection is denoted by the blue box 


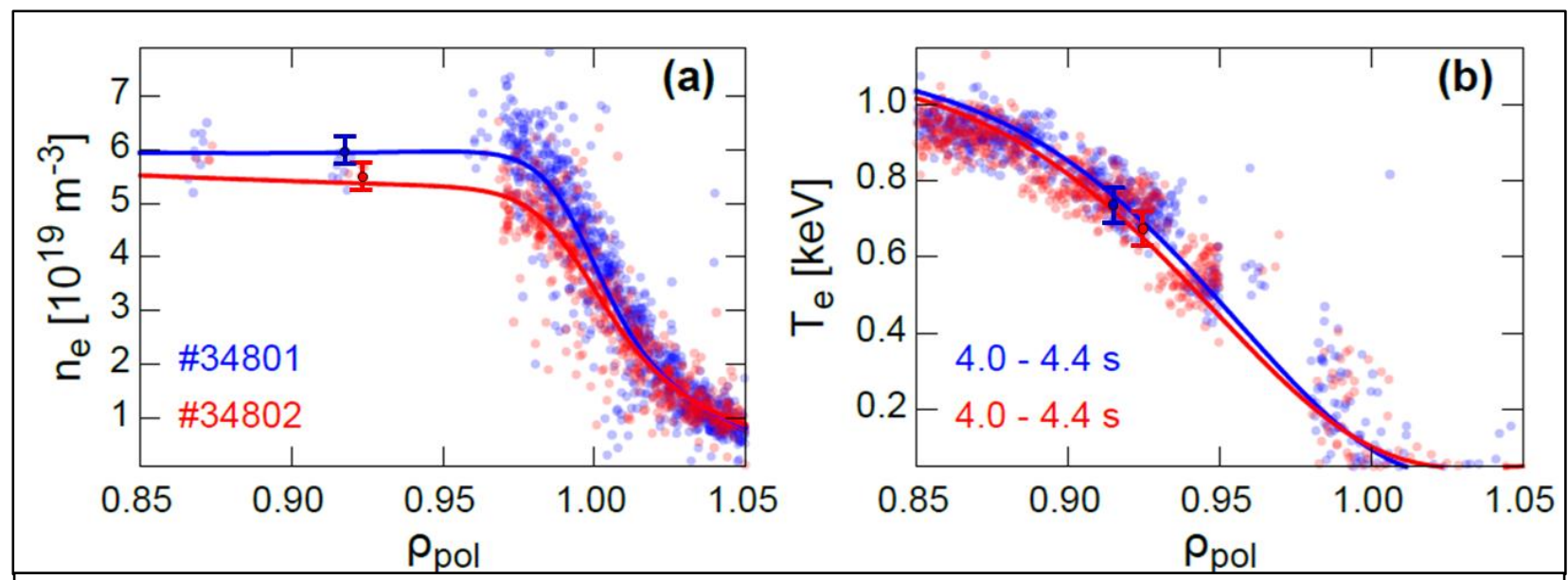

Figure 7 : Comparison of changes in electron temperature and density profiles as a result of boron nitride powder injection. Discharge AUG 34801 containing BN injection is compared to AUG 34802 which was performed with the same shaping and input powers but without powder injection showing that the increases in discharge stored energy are caused by an increae to the density pedestal. The electon density shown in panel a) combines the lithium beam measurement with Thomson scattering along with a modified Tanh fit to generate the profile. The electron density in panel b) is a combination of the Electron Cyclotron Emission measurement and Thosmson scattering data. Respective error bars are shown in both graphs at $\rho \sim 0.92$. 


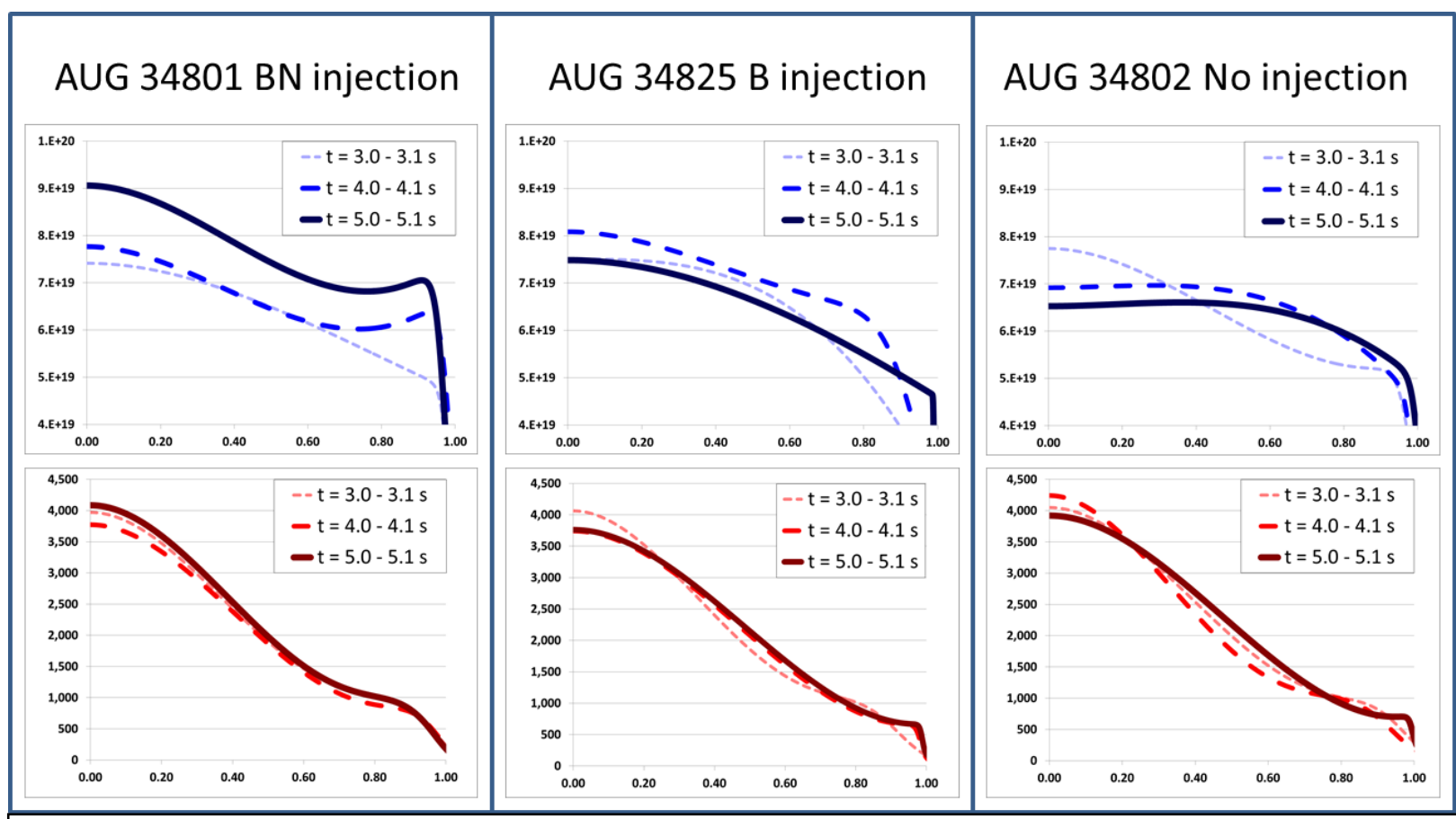

Figure 8 : Full electron density (upper) and temperature (lower) profile evolutions for discharges with BN, B and No injection generated the same way as figure 7 with similar error, and data points omitted for clarity. For discharges with injection, the $t=3.0-3.1$ average is prior to the injection of material, while the later times are during mass injections. The only significant change to the discharges observed during the course of injection is to AUG34801 which involved injection of boron nitride and subsequent pedestal improvements. Note that the zero has been suppressed in the density traces to allow better resolution of the change in profile density with density expressed in $\mathrm{m}^{-3}$ and temperature expressed in $\mathrm{eV}$ with a $\rho_{\text {pol }}$ horizontal axis. 


$\begin{aligned} & \text { Phot }=34802 \\ & \text { Prad tot }=5.8202 \mathrm{MW} \\ & \text { Prad sep }=2.0433 \mathrm{MW} \\ & \text { Prad div }=1.7616 \mathrm{MW}\end{aligned} \mid-12$




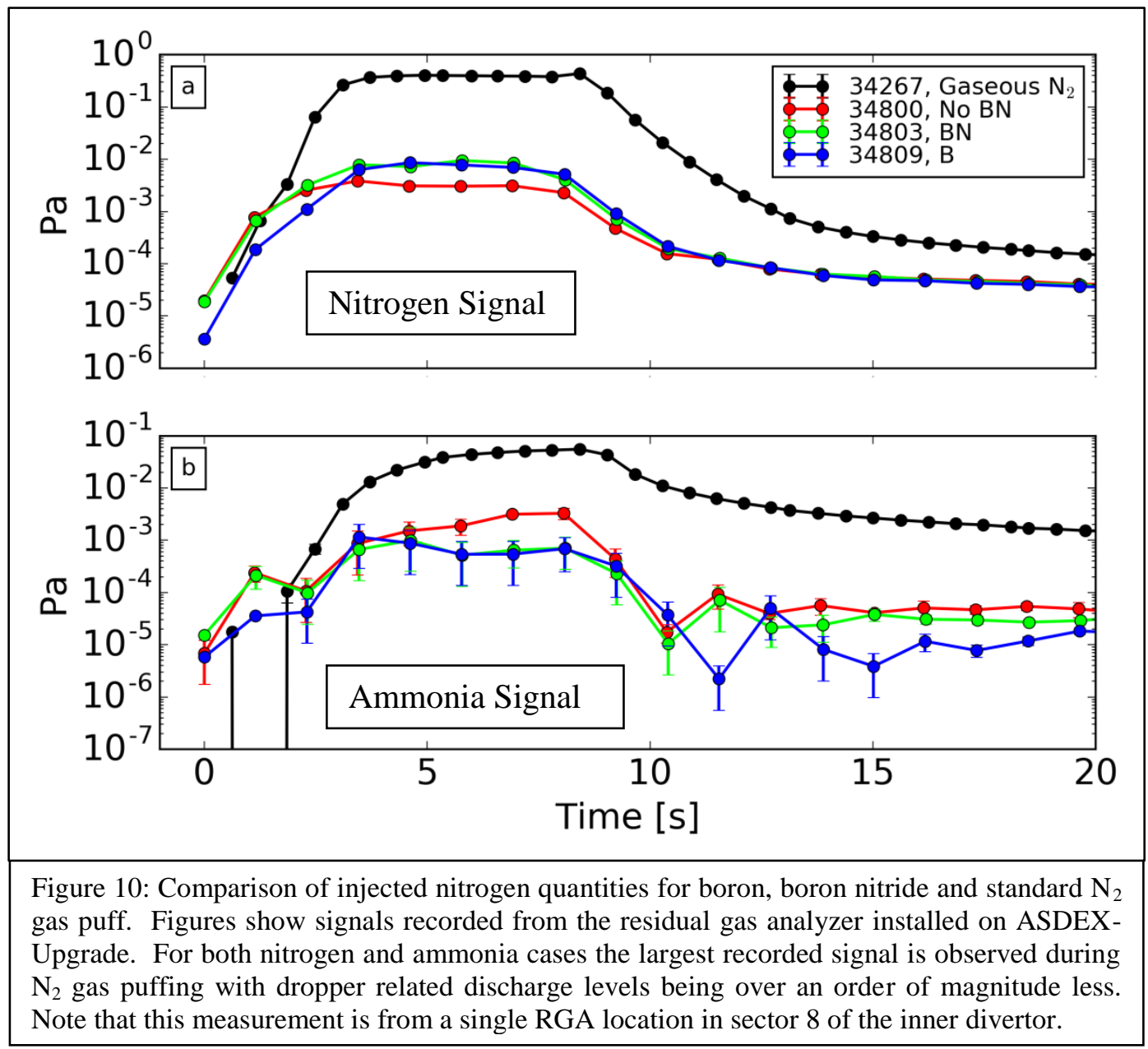




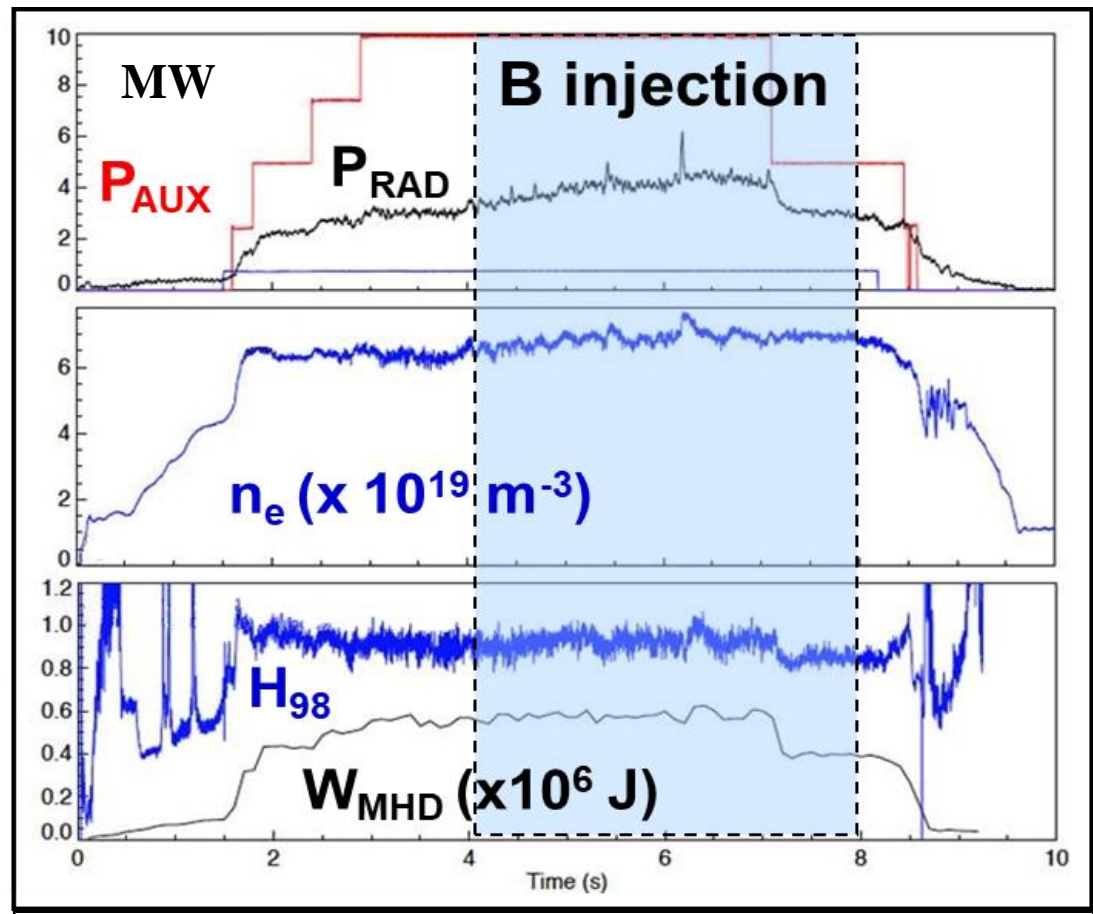

Figure 11: Effects of boron injection on overall plasma parameters for discharge \#34809. The introduction of boron particulates into the discharge has a small effect on the density and creates a $40 \%$ increase in the radiated power, however the effects on confinement as evidenced by the $\mathrm{H}_{98}$ and $\mathrm{W}_{\text {MHD }}$ traces are minimal. 

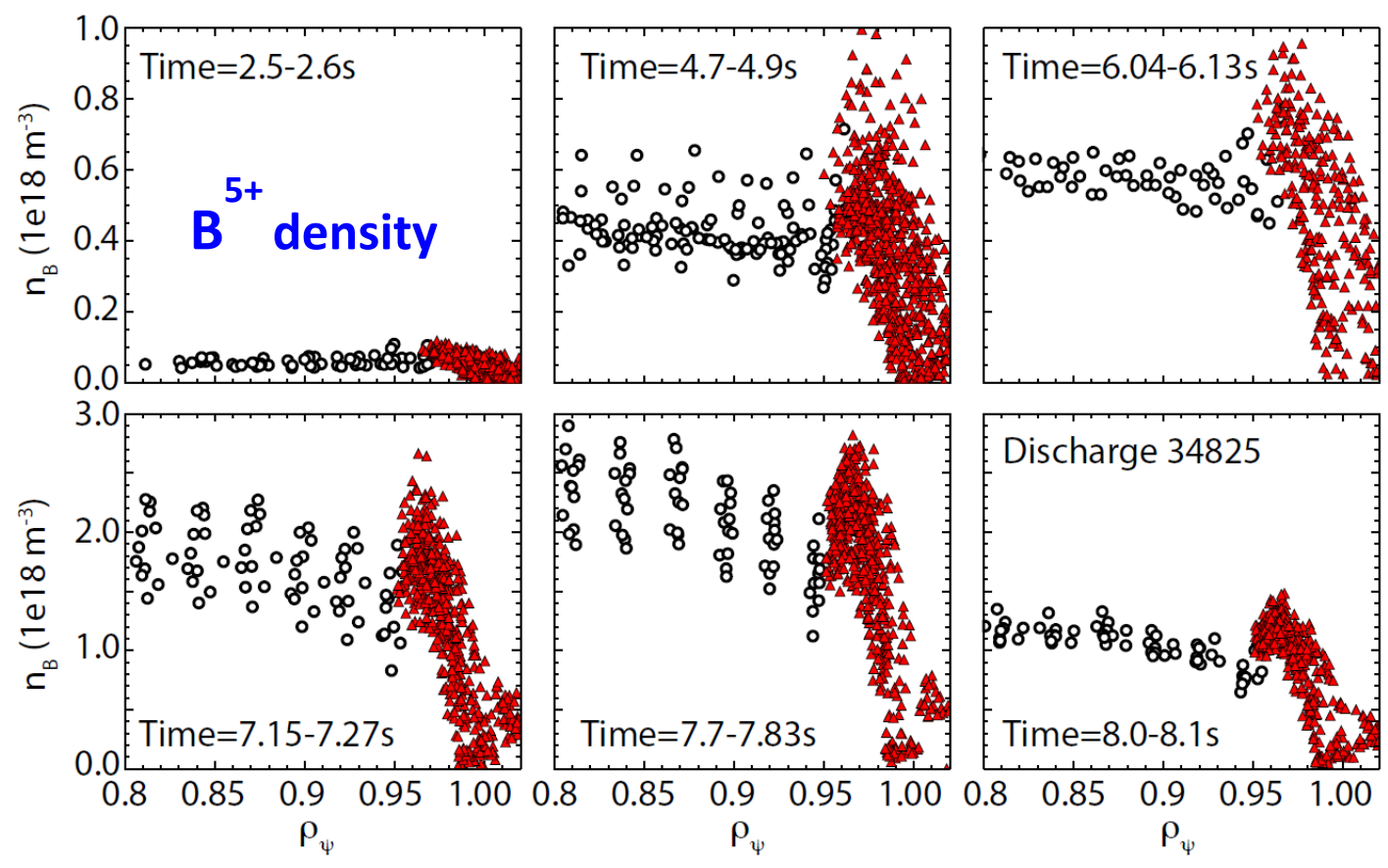

Figure 12: Boron impurity concentrations during powder dropper application. The above graphs show the edge and core concentrations of boron V during ASDEX discharge 34815. The filled triangles are data from the CMR diagnostic, while the open circles represent data from the CER charge exchange system with the mismatch at diagnostic crossover the result of a calibration issue The scatter seen in these measurements is a result of the rapid discharge evolution due to irregular injection of B powder and not indicative of measurement error. In addition, note that the vertical scale is a factor of 3 larger for the lower set of 3 graphs. 


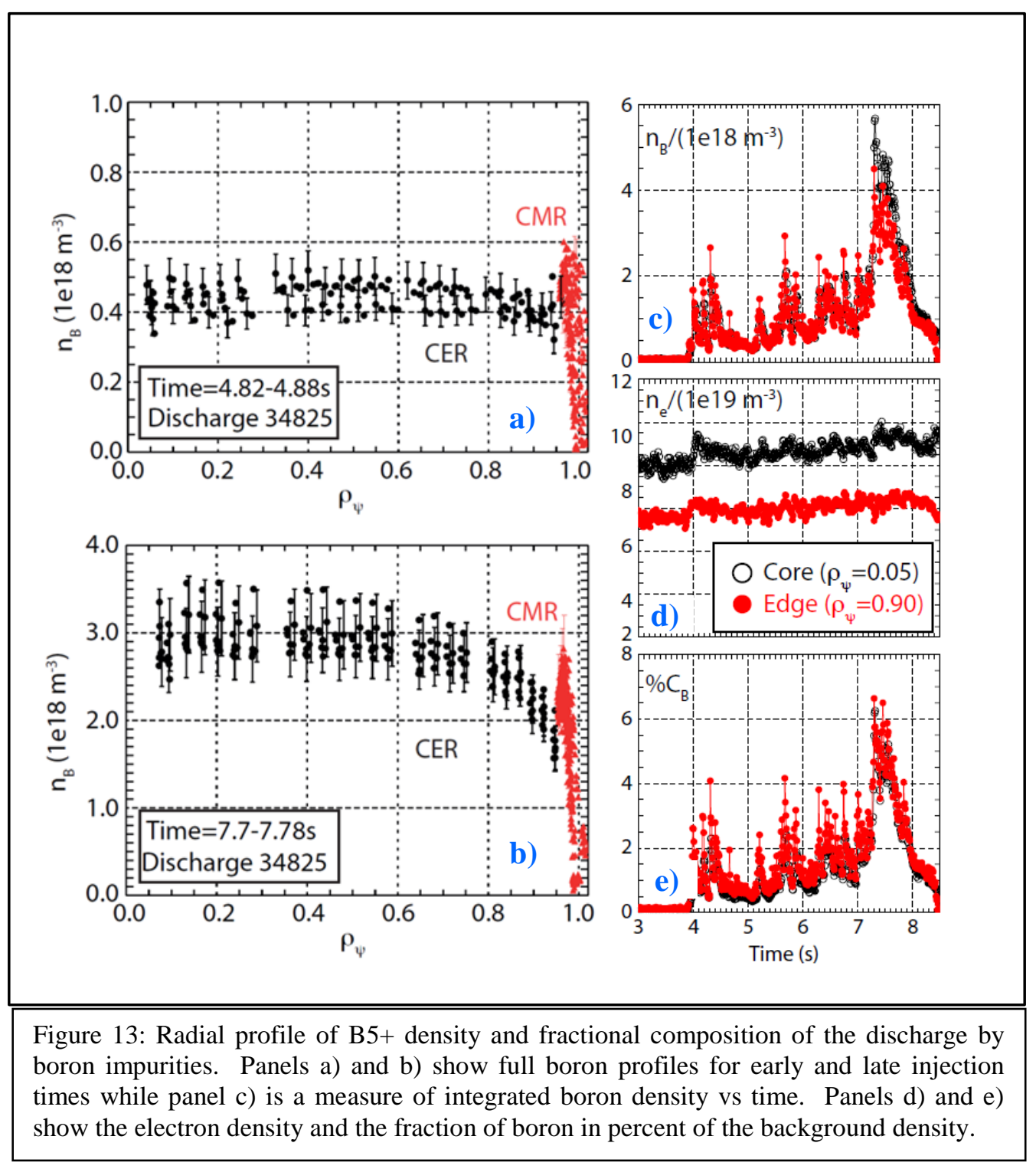




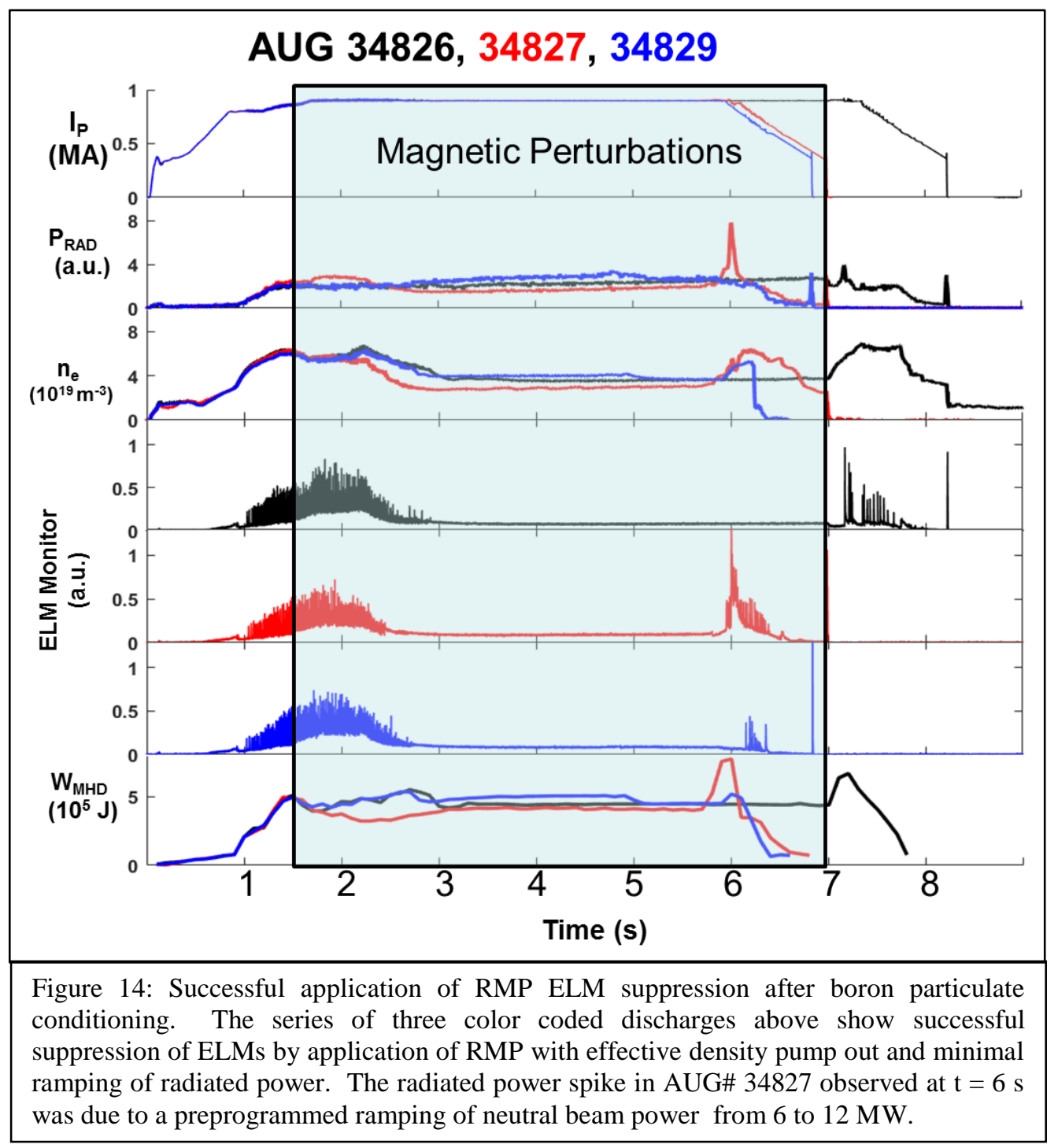




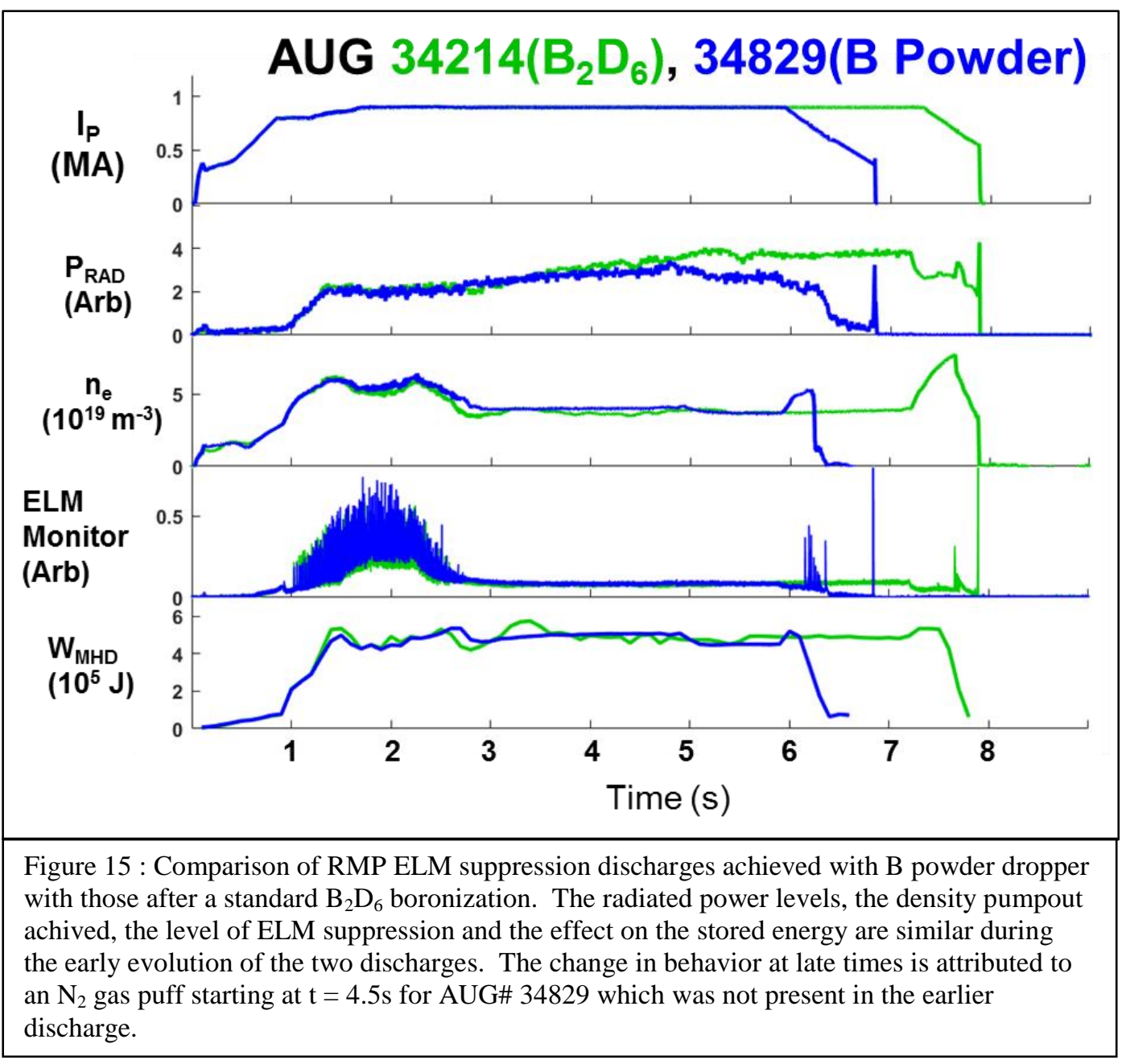

